\title{
Time to Antibiotics and Hospital Length of Stay in Emergency Department Patients Admitted for Pneumonia: A Retrospective, Cross-Sectional Analysis
}

\author{
Hany Y Atallah MD*, Dilani Weerasuriya MD, Jeremy Hess MD MPH, Debra Houry MD MPH, \\ Daniel Wu MD, Leon L Haley Jr., MD, MHSA
}

\author{
Department of Emergency Medicine, Emory University School of Medicine, Atlanta, 30303, Georgia, United States
}

*Corresponding Author: hatalla@emory.edu

Copyright (C) 2013 Horizon Research Publishing All rights reserved

\begin{abstract}
Background: Studies have reported mixed findings regarding timing of antibiotic delivery in patients admitted for pneumonia and clinical outcomes. We hypothesized that there is no association between the time to antibiotics and the hospital length of stay (LOS) for patients admitted from the emergency department (ED) to the hospital for pneumonia.Objective: To compare the hospital LOS of patients admitted from the ED to the hospital for pneumonia based on the interval between ED arrival and first dose of antibiotics categorized to four hours or less, four to six hours, and greater than six hours.Methods: We conducted a retrospective chart review at a single inner city ED on patients who had admitting and discharge diagnoses of pneumonia and received antibiotics in the ED over a 17 month period. Using structured abstraction sheets, a trained reviewer identified cases and abstracted data on hospital LOS, time of arrival to first antibiotic dose, admission bed type, and other patient factors. The primary outcome was hospital LOS. Data were analyzed using descriptive statistics, ANOVA, and linear regression.Results: Nine hundred ninety ninecases were identified and541 met inclusion criteria. Four hundred eighty three (89\%) were African American, 240 (44\%) were female, the average age was 52 years old (SD 15), and 186 (34\%) were HIV positive.There was no significant difference in LOS based on time to antibiotics. Controlling for patient factors including pneumonia type (community or health care associated) did not result in a significant difference in LOS across the time to antibiotic categories.Conclusions: In this population of pneumonia patients admitted to the hospital from the ED, time to first antibiotic dose was not significantly correlated with hospital LOS. Controlling for demographics and disease severity did not affect the observed relationship.
\end{abstract}

Keywords Pneumonia, Length of Stay, Antibiotics

\section{Introduction}

Pneumonia accounts for significant morbidity and mortality in the United States, with 2-4 million cases annually. ${ }^{1}$ Though antibiotics are the most effective treatment of bacterial pneumonia and intuition suggests that early antibiotic administration would result in improved outcomes, the relationship between the time to antibiotic administration for patients admitted from the emergency department (ED) and indicators of morbidity and mortality remains unclear. ${ }^{2,3} \mathrm{~A}$ landmark study by Battleman and colleagues reviewed 700 patients treated for community acquired pneumonia (CAP) and found that antibiotic administration within 8 hours of presentation could significantly decrease length of stay (LOS) in the hospital. ${ }^{4}$ This conclusion supported the Joint Commission on Accreditation of Healthcare Organization's (TJC) and the Centers for Medicaid and Medicare Service's (CMS) regulations at the time that patients diagnosed with CAP receive antibiotics within 8 hours of presentation. Since then, TJC/CMS regulations have varied from antibiotics within four hours to six hours.

The Inpatient Prospective Payment System (IPPS) updates for 2011 state that for the fiscal year 2014 and subsequently, the time to receipt of initial antibiotics will no longer be part of the Hospital Inpatient Quality Reporting Program measure set. It also states that starting with January 1, 2012 discharges, hospitals no longer will be required to submit data on these measures. However, many hospitals continue to report 6 hour calculations, ${ }^{5}$ and efforts to maximize compliance with the rules remain a significant administrative consideration. ${ }^{6}$

Recent research exploring both intended and unintended consequences of these guidelines suggests they may need to be revisited. Cheng et al found that patients who died during hospitalization had a shorter time to antibiotics and did not find a relationship between LOS and antibiotic timing. ${ }^{7}$ Welker et al demonstrated that reducing the goal 
time to first antibiotic dose from within 8 hours to within 4 hours increased the discrepancy between the ED physician's admitting diagnoses and the discharge physician's diagnoses by $8 \% .{ }^{8}$ The reduction in required time to antibiotics resulted in physicians over-diagnosing pneumonia so as to not miss the mandated time to treatment window. These different regulations may not only be confusing but also increase antibiotic dispersal to patients in whom pneumonia will not be the final diagnosis, potentially increasing antibiotic resistance and adverse drug reactions.

To evaluate whether time to antibiotics was significantly associated with outcomes in our institution we devised a study to evaluate whether time to antibiotics was associated with hospital LOS in a sample of pneumonia patients admitted from the ED. We focused on the six hour threshold emphasized in the most recent recommendation for patient arrival to antibiotics. Our study objective was to determine if delays in antibiotics over six hours resulted in longer hospital LOS

\section{Materials and Methods}

\subsection{Study Design}

During the time of the study, the hospital medical record system was paper-based. We performed a retrospective chart review on patients admitted from the ED with a diagnosis of pneumonia. One of the authors with previous training abstracted the data from patient's discharge summaries using an electronic form. Only one person abstracted the data and inter-rater reliability was not measured. The research team met weekly to discuss any discrepancies or questions that had arisen during the data abstraction. Emory University Institutional Review Board approved the study and determined the study was exempt.

\subsection{Study Setting and Population}

The study was conducted in a large, urban, county hospital in the Southeast with approximately 28,000 annual admissions and 400,000 non-emergency outpatient visits that serves as a primary teaching hospital for two medical schools. The hospital serves a largely African American and medically indigent population. The ED is the largest Level One Trauma Center for the region and during the study period treated approximately 145,000 patients. The ED is staffed by attending physicians from one of the two university programs as well as emergency medicine and rotating residents.

\subsection{Selection of Participants}

We defined a case as an adult ED patient (aged 18 years or older)seen between December 2008 to April 2010 inclusive who was admitted to the hospital with both an admission and discharge diagnosis of pneumonia. Cases were selected from a continuously updated spreadsheet of ED patients who were suspected of having pneumonia that was maintained in order to track compliance with TJC regulations. We pulled charts on these cases to identify potential cases. Exclusion criteria included: leaving against medical advice at any point during the hospital stay; age less than 18 at the time of admission; receipt of antibiotics within seven days prior to hospital admission; death during admission; and prolongation of hospital admission for a problem other than the patient's pneumonia, e.g. the workup of a new cancer diagnosis. Prolongation of hospital admission for another reason was determined by directly reviewing the discharge summary and hospital course to determine if a delay was mentioned.

\section{Methods of Measurement}

Information on the following patient factors was abstracted: time of ED arrival, time of first antibiotic administration, and use of the ED's pneumonia pathway. The ED pneumonia pathway was developed to help quickly identify and treat patients who were at risk of having pneumonia. This was primarily based on the patient's triage chief complaint and vital signs. We obtained additional demographic data, including age, gender, race, and admission bed type (floor, step-down, intensive care) from the patient face sheet. From the discharge summary we abstracted admission diagnoses, discharge diagnoses , hospital LOS(defined by time between admission date and discharge date), diagnosis of community acquired pneumonia versus hospital acquired pneumonia, vital signs and symptoms at presentation, alcohol/tobacco/illicit substance habits, and premorbid conditions such as hypertension, diabetes, positive HIV status, congestive heart failure, asthma, an active cancer, dementia, coronary artery disease, psychiatric illness, and history of cerebrovascular accident. Missing information was coded as absent but cases were still included in the study as long as there was data for time to first dose of antibiotics, hospital LOS, and admission bed type.

Diagnosis of community acquired pneumonia versus healthcare associated pneumonia (HCAP) was determined by which was listed in the discharge diagnoses and discharge summary. This study did not attempt to capture inpatients that developed pneumonia in the hospital. Patients with HCAP were identified upon presentation to the ED and were questioned to determine if they met the diagnostic criterion for HCAP. The admission bed type (floor, step-down, intensive care) was used as a proxy for the severity of the patient's illness at presentation.

\section{Data Analysis}

Data were analyzed using descriptive statistics, ANOVA, and linear regression. Mean hospital LOS was stratified by time to antibiotic category ( $<4$ hours, $4-6$ hours, $>6$ hour) and 
category means were compared using ANOVA. Linear regression was used to analyze LOS as a continuous variable based on time to antibiotic category and controlling for patient characteristics. Regression analyses were done for the entire sample as well as within presentation severity categories, and moderate and severe cases were combined to increase statistical power. A secondary analysis using linear regression was conducted on a subset of cases that were HIV negative, aged $>65$, and had discharge diagnosis of community acquired pneumonia. Data were analyzed using SPSS version 16.0. Regression model dummy coding was used for the variables time to treatment, severity, and HIV diagnosis.

\section{Results}

One thousand seventy patient visits were initially flagged as ED pneumonia cases during our study period. Of these,
999 had charts available for review, and of those 541 cases met our inclusion criteria. 458 patients were excluded from the study because their final diagnosis was not pneumonia, they had received antibiotics in the previous 7 days, they left AMA or they died during hospitalization. Six patients died from CAP/HCAP, 11 left AMA with a primary discharge diagnosis of CAP/HCAP and 132 patients had a different primary diagnosis. Of those 132, 3 left AMA and 15 died.

Of the 541 cases, $89 \%$ were African American, 44\% were female, and the average age was 52 (SD 15). See Table One for further description of sample demographics. The average hospital LOS for cases who received antibiotics in less than 4 hours was 5.8 days, 4-6 hours 6.2 days, and $>6$ hours 4.9 days. Forty five percent of cases received antibiotics during their ED stay in less than 4 hours, $27 \%$ in 4-6 hours, and $28 \%$ in over six hours. There was no significant difference in hospital LOS by time to antibiotic category. See Tables 1 and 2 for further details.

Table 1. Patient Characteristics

\begin{tabular}{|c|c|c|c|c|}
\hline \multirow{2}{*}{ Characteristics } & \multicolumn{3}{|c|}{ Time to First Antibiotic Dose } & \multirow{2}{*}{$\begin{array}{c}\text { All } \\
\text { Patients }\end{array}$} \\
\hline & Within 4 Hours & 4 to 6 Hours & After 6 Hours & \\
\hline Total & 244 & 146 & 151 & 541 \\
\hline Race & $\begin{array}{c}\text { African America: } 218 \\
(89 \%) \\
\text { Caucasian: } 15(6 \%)\end{array}$ & $\begin{array}{c}\text { African American: } 128 \\
(88 \%) \\
\text { Caucasian: } 8(5 \%)\end{array}$ & $\begin{array}{c}\text { African American: } 137 \\
\text { (91\%) } \\
\text { Caucasian: } 11(7 \%)\end{array}$ & $\begin{array}{c}\text { African American: } 483 \\
(89 \%) \\
\text { Caucasian:34 } \\
(6 \%) \\
\end{array}$ \\
\hline Gender & Female: $110(45 \%)$ & Female: $58(40 \%)$ & Female: $72(48 \%)$ & $\begin{array}{l}\text { Female: } 240 \\
\quad(44 \%)\end{array}$ \\
\hline Age (SD) & $51(15)$ & $54(14)$ & $52(17)$ & $52(15)$ \\
\hline HIV Positive & $93(39 \%)$ & $48(33 \%)$ & $45(30 \%)$ & $186(34 \%)$ \\
\hline $\begin{array}{l}\text { Median Length of Stay in } \\
\text { Days }\end{array}$ & 4 & 4 & 3 & 4 \\
\hline $\begin{array}{c}\text { Mean Length of Stay in } \\
\text { Days }\end{array}$ & 5.8 & 6.2 & 4.9 & 5.7 \\
\hline
\end{tabular}

Table 2. Severity of Patients within the Different Time to Treatment Groups

\begin{tabular}{|c|c|c|c|c|c|}
\hline \multicolumn{5}{|c|}{ Time to First Antibiotic Dose (\% of total cases) } \\
\hline \multirow{3}{*}{ Severity } & Within 4 Hrs & $4-6$ Hrs & \multicolumn{2}{|c|}{ After 6 Hrs } & Total \\
\cline { 2 - 6 } & Mild & $196(36 \%)$ & $132(24 \%)$ & $141(26 \%)$ & $469(87 \%)$ \\
\cline { 2 - 6 } & Intermediate & $31(6 \%)$ & $9(2 \%)$ & $9(2 \%)$ & $49(9 \%)$ \\
\cline { 2 - 6 } & Severe & $17(3 \%)$ & $5(1 \%)$ & $1(0 \%)$ & $23(4 \%)$ \\
\cline { 2 - 6 } & Total & $244(45 \%)$ & $146(27 \%)$ & $151(28 \%)$ & $541(100 \%)$ \\
\hline
\end{tabular}


Time to Antibiotics and Hospital Length of Stay in Emergency Department Patients Admitted for Pneumonia: A Retrospective, Cross-Sectional Analysis

Table 3. Average Length of Stay within the Different Time to Treatment Groups

\begin{tabular}{|c|c|c|c|}
\hline \multicolumn{4}{|c|}{$\begin{array}{c}\text { Mean Time to First Antibiotic Dose (cases) } \\
\mathrm{p}>0.05 \text {, ANOVA for All Cases }\end{array}$} \\
\hline & Within 4 Hrs & $4-6$ Hrs & After 6 Hrs \\
\hline $\begin{array}{c}\text { Only Mild Cases (Total = 469) } \\
(\text { Total = 49) }\end{array}$ & 4.9 Days (196) & 5.6 Days (132) Days (141) \\
\hline $\begin{array}{c}\text { Only Intermediate Cases } \\
\text { Only Severe Cases (Total = 23) }\end{array}$ & 7.8 Days (31) & 8.8 Days (9) & 2.7 Days (9) \\
\hline All Cases (Total = 541) & 12.2 Days (17) Days (1) \\
\hline $\begin{array}{c}\text { Cases with age }>65, \text { HIV Negative, with a diagnosis of CAP } \\
(\text { Total = 67) }\end{array}$ & 5.8 Days (244) & 6.2 Days (146) & 4.9 Days (151) \\
\hline
\end{tabular}

Since the severity of the cases ranged from mild to severe (based on admission bed type) within in the three timing groups, we performed a linear regression comparing each of these groups. There was no difference in the hospital LOS between the three severity groups when adjusted for door to antibiotic time, $\mathrm{p}<0.05$. See Table 3 .

Mild cases were separated into the three time to treatment categories. There was no statistically significant difference in the $<4$ hour (4.9 day LOS), 4-6 hour (5.6 day LOS), and $>6$ hour (5.0 day LOS) treatment groups. Intermediate cases were also separated into the three times to treatment categories. There was no statistically significant difference in the $<4$ hour (7.8 day LOS), 4-6 hour (8.8 day LOS), and $>6$ hour (4.2 day LOS). The LOS for severe cases was also compared. Again, there was no statistically significant difference between the $<4$ hour treatment group (12.2 days) and the 4-6 hour treatment group (17.6 day LOS). We did not compare these groups to the severe case that received antibiotics in $>6$ hours since there was only one case in that group.

In our secondary analysis of older $(>65)$, HIV negative patients with CAP $(n=67), 29$ cases $(43.3 \%)$ received antibiotics with 4 hours, $19(28.4 \%)$ between 4 to 6 hours, and $19(28.4 \%)$ after 6 hours. The $<4$ hour group averaged a 5.3 day length of stay, the 4-6 hour group a 5.2 day stay, and the $>6$ hour group a 4.6 day stay. The difference between the three groupswas not statistically significant.

\section{Limitations}

Our study had several limitations. The population is relatively homogeneous in terms of race and socioeconomic status. LOS had to be recorded in days rather than hours because the electronic financial system only recorded daily timestamps. Since we compared mean LOS, values may have been skewed by outliers. Also, cases were included if they had a final diagnosis of pneumonia, however, many cases had several other final diagnoses, some of which were listed before pneumonia. It is unclear as to whether LOS in such cases was a reflection of their pneumonia process, one of their other diagnoses, or particular diagnostic constellations and syndromes.

It may be difficult to compare the findings of our primary analysis with other studies as we did not initially restrict our population by age, HIV status or HCAP diagnosis, despite other studies doing so, because TJC/CMS did not restrict recommended time to antibiotic dosing by these criteria. However, our secondary analysis suggests that time to antibiotics was not significantly associated with LOS among a subset of patients. In addition, the sample size of the study group was small as this was a pilot study. Lastly, our results may not be generalizable to other settings given the particular population served by the hospital where we conducted the study.

\section{Discussion and Conclusion}

In this study we found that there was no significant relationship between the time to first dose of antibiotics and hospital LOS in patients admitted from the ED for pneumonia. Controlling for severity of presentation did not affect the significance of the relationship.The only relationship that we found to be significant was that cases admitted to higher acuity beds had longer hospital LOS. This is a predictable finding, because it is to be expected that sicker patients would have longer hospital stays.

This study is relatively unique because of our urban patient population. The urban population is not limited to our study setting, as many underserved communities have similar populations, but few are the subject of pneumonia treatment studies.

TJC regulations are based on studies of specific populations, e.g. patients aged 65 or older and HIV negative. These characteristics only apply to $12 \%$ of the patients admitted for pneumonia during the study period. Even when these same restrictions were placed on our patient population, no significant relationship was found between antibiotic timing and hospital LOS.

Previous studies have shown that patients are inappropriately being diagnosed with bacterial pneumonia 
and receiving antibiotics for viral illnesses on an alarming basis. ${ }^{9-12} \mathrm{We}$ believe that those results, in combination with our own findings, indicate that additional research on the impact and efficacy of antibiotic timing is needed. This research should target a broader demographic range and consider the potential implications of four hour, six hour, and other antibiotic guidelines on practice patterns, inappropriate antibiotic administration, unnecessary medication costs, and likelihood of antibiotic resistance in addition to impacts on mortality for pneumonia patients.

\section{Conflicts of Interests}

Dr. Dilani Weerasuriya conducted this work while a medical student at Emory University School of Medicine. She actively participated in the design of the study, acquisition of data, analysis and interpretation of the data, drafting of the manuscript, and critical revisions. Dr. Hany Atallah supervised the conception and design of the study, the analysis and interpretation of the data, the drafting of the manuscript and critical revisions. Drs. Atallah and Weerasuriya had full access to all of the data in the study and take responsibility for its integrity and accuracy. None of the investigators has potential conflicts of interests to disclose, including specific financial interests, relationships or affiliations relevant to the subject matter or materials discussed in the manuscript.

\section{REFERENCES}

[1] Jacobs MR. Antimicrobial-resistant Streptococcus pneumonia: trends and management. . Expert Rev Anti Infect Ther. 2008;6:619-35.

[2] Nazarian DJ, Eddy OL, Lukens TW, Weingart SD, Decker WW. Clinical policy: critical issues in the management of adult patients presenting to the emergency department with community-acquired pneumonia. Annals of Emergency Medicine. 2009 Nov;54(5):704-31.
[3] Yu KT, Wyer PC. Evidence-based emergency medicine/critically appraised topic. Evidence behind the 4-hour rule for initiation of antibiotic therapy in community-acquired pneumonia. Annals of Emergency Medicine. 20082008 May;51(5):651-62.

[4] Battleman D, Callahan M, Thaler H. Rapid Antibiotic Delivery and Appropriate Antibiotic Selection Reduce Length of Hospital Stay of Patients with Community-Acquired Pneumonia. Arch Intern Med. 2002;162:682-8.

[5] Centers for Medicare and Medicaid Services. Hospital Inpatient Prospective Payment Systems for Acute Care Hospitals and the Long-term Care Hospital Prospective Payment System and FY 2012 Rates; Hospitals' FTE Resident Caps for Graduate Medical Education Payment. Washington, DC2011.

[6] Hill PM, Rothman R, Saheed M, Deruggiero K, Hsieh Y-H, Kelen GD. A comprehensive approach to achieving near 100\% compliance with the Joint Commission Core Measures for pneumonia antibiotic timing. American Journal of Emergency Medicine. 2011 Nov;29(9):989-98.

[7] Cheng A, Buising K. Delayed Administration of Antibiotics and Mortality in Patients with Community-Acquired Pneumonia. Ann Emerg Med. 2009;53:618-24.

[8] Welker J, Huston M, McCue J. Antibiotic Timing and Errors in Diagnosing Pneumonia. Arch Intern Med. 2008;168:351-6.

[9] Stone S, Gonzales R, Maselli J, Lowenstein S. Antiobiotic prescribing for patients with colds, upper respiratory tract infections, and bronchitis: a national study of hospital-based emergency departments. Annals of Emergency Medicine. 2000;36:320-7.

[10] Gilbert E, Lowenstein S, Koziol-McLain J, Barta D, Steiner J. Chart Reviews in Emergency Medicine: Where are the Methods? . annals of Emergency Medicine. 1996;27:305-8.

[11] Kanwar M, Brar N, Khatib R, Fakih MG. Misdiagnosis of Community-Acquired Pneumonia and Inappropriate Utilization of Antibiotics*Side Effects of the 4-h Antibiotic Administration Rule. CHEST Journal. 2007;131(6):1865-9.

[12] Mitka M. TJC Tweaks Emergency Departments' Pneumonia Treatment Standards. JAMA. 2007;297:1758-9. 